\title{
Reconfigurable Propulsion Systems for the inspection of off-shore power generation plants
}

\author{
Benedetto Allotta \\ Dept. of Industrial Engineering \\ University of Florence \\ Florence, Italy \\ allotta.benedetto@unifi.it \\ Marco Pagliai \\ Dept. of Industrial Engineering
}

\author{
University of Florence \\ Florence, Italy \\ m.pagliai@unifi.it \\ Luca Pugi \\ Dept. of Industrial Engineering \\ University of Florence \\ Florence, Italy \\ luca.pugi@unifi.it
}

\begin{abstract}
Inspection of offshore plants or harsh environments, require underwater vehicles with high degree of autonomy, performances and maneuverability. These characteristics are deeply influenced by the features of propulsion layouts of this kind of vehicle. In particular, the study of maneuverability required by ROV and AUV implies the modelling of four quadrants operation of propellers and of threedimensional interactions arising between complex propulsion and hull geometries. In this work, an application of reconfigurable propulsion layout of an inspection vehicle for offshore operation or harsh environments is introduced.
\end{abstract}

Keywords-Autonomous underwater vehicle, propulsion magnetic transmission systems, fast prototyping, optimized motor design, oil-pressure compensated actuators.

\section{INTRODUCTION}

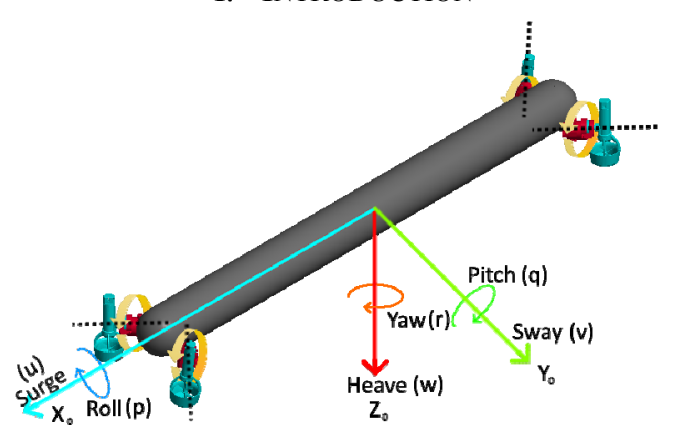

Figure 1 Example of application with four orientable thrusters with SNAME notation.

In this work, the applicability of a configurable propulsion layout for underwater vehicles for offshore operation will be investigated. The proposed layout is based on an array of low cost pivoted thrusters that can be easily customized and optimized with respect to operating conditions and mission profiles, visible in the scheme of Figure 1. In particular, in order to optimize cost, encumbrances and maintenance, authors developed a solution so that the orientation of each thruster could be calibrated with respect to a single pivoting axis.
In existing solutions available in literature such as Smart E AUV, [1], [2], three pivoted thrusters are used to perform a holonomic control of the six degree of freedom of the AUV.

In this study, authors want to use four pivoting actuators in order to control the vehicle motion by improving the maneuverability and efficiency with respect to traditional AUVs.

In details, the work is organized as follows:

- Current State of the Art and definition of a benchmark vehicle and operating scenario.

- Preliminary Design of an Actuator unit according to chosen benchmark. Execution of preliminary tests and the development of simplified models, adopted to identify main features of the prototype in terms of performances and efficiency.

- Development of a Virtual Model of the whole system with the aim of investigating the potential features of the proposed approach.

\section{STATE OF ART}

This work is based on the experience acquired by authors in the prototyping of hybrid multi-role AUVs (Autonomous Underwater Vehicles) TIFONE [3], [4], [5] and MARTA [6], [7], whose propulsion layout is visible in Figure 2. In these vehicles, two rear propellers are used for standard-straight navigation and a certain number of tunneled thrusters are devoted to control orientation or to keep the vehicle hovering over an assigned target. This kind of motor layout involve the use of high number of controlled independent actuators ( at least six). In order to reasonably reduce costs and to increase modularity and reliability of the whole system fixed pitch propellers, which are almost identical for all actuated axis, are usually adopted.

The resulting propulsion layout makes possible the control of five degree of freedom, which are described according the classical SNAME notation, widely adopted in literature [8]: 
- Surge: longitudinal load $\mathrm{X}$ is the sum of thrusts delivered by the two rear propellers.

- Sway and heave: lateral load Y and Z are respectively the sum of thrusts of the two lateral and vertical tunnel thrusters.

- Pitch and Yaw rotations: vertical and lateral thrusters respectively control these rotations. A remarkable point is the Yaw actuation. Indeed, this rotation has a redundant actuation, since a rotating torque $\mathrm{N}$ can be also produced using the two rear propellers.

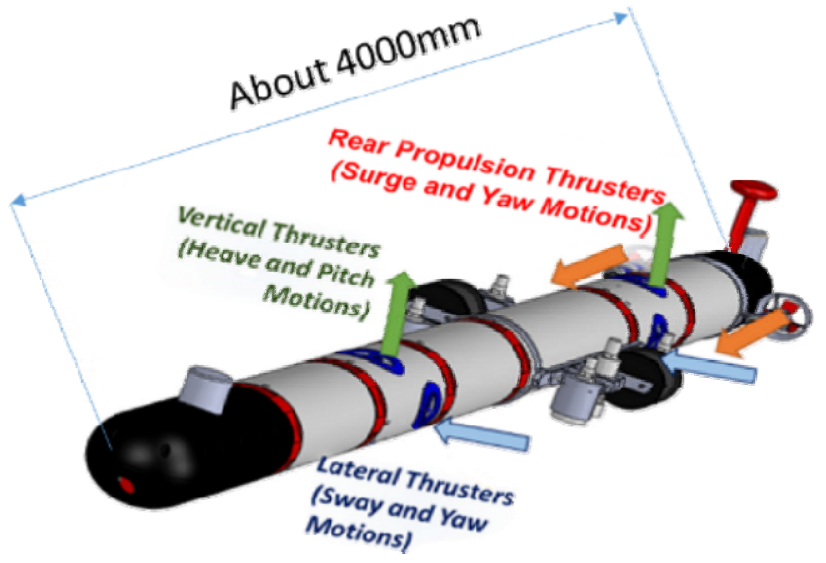

Figure 2 MARTA AUV propulsion system layout and corresponding encumbrances.

Many existing AUVs adopt similar combinations of fixed pitch rear propellers and lateral tunnel thrusters in order to obtain a good vehicle maneuverability. It is possible to cite many examples, such as C-Scout [9], Remus [10], Proteus [11], Delphin2 [12] and Folaga [13].

In these kind of layouts, the actuation of different degree of freedom is highly decoupled, making it quite easy the control of the vehicle. In addition, a wise choice of the propeller rotation sense can reduce motion disturbances arising from propellers reaction torques.

An easy controllability is an important requirement also for the design of commercial ROVs (Remotely Operated underwater Vehicles), where the vehicle has to be maneuvered by a human operator, with a limited level of additional automation. Some examples of propulsion layouts often adopted on ROVs are visible in Figure 3 and in TABLE I.

Unfortunately, one of the drawbacks of the propulsion layout adopted on MARTA is the encumbrances of the propulsion system with respect to the payload. As visible in Figure 2, the length of vehicle is about $4000 \mathrm{~mm}$ (about 18 times bigger with respect to the hull diameter). However, the total length of the three propulsion modules is more than $1.2 \mathrm{~m}$, so a reduction of these elements can result in a significant length reduction. In addition, although the motor layout, adopted on MARTA has a redundant configuration, does not allow the controll of the roll angle. More generally, it should be noticed that the over-cited propulsion layouts could not be dynamically reconfigured during the mission so their usage could be critical in scenarios with uncertain operating parameters such as water density, currents, unexpected mission profile, availability or reliability of one or more actuators.

TABLE I. CONTROLled AND UNCONTROLLED DEGREE OF FREEDOM FOR SOME TYPICAL PROPULSION LAYOUTS ADOPTED BY COMMERCIAL ROVS.

\begin{tabular}{|c|c|c|c|c|c|c|}
\hline & Surge & Sway & Heave & Roll & Pitch & Yaw \\
\hline a) $* * *$ & $\mathrm{C}^{*}$ & $\mathrm{C}^{*}$ & $\mathrm{C}^{*}$ & $\mathrm{C}^{*}$ & $\mathrm{NC}^{* *}$ & $\mathrm{C}^{*}$ \\
\hline b) $* * *$ & $\mathrm{C}^{*}$ & $\mathrm{C}^{*}$ & $\mathrm{C}^{*}$ & $\mathrm{NC}^{* *}$ & $\mathrm{NC}^{* *}$ & $\mathrm{C}^{*}$ \\
\hline c) $* * *$ & $\mathrm{C}^{*}$ & $\mathrm{C}^{*}$ & $\mathrm{C}^{*}$ & $\mathrm{NC}^{* *}$ & $\mathrm{NC}^{* *}$ & $\mathrm{C}^{*}$ \\
\hline \multicolumn{7}{|c|}{$\begin{array}{ll}\mathrm{C}^{*}=\text { controlled D.O.F. } & \text { NC**uncontrolled D.O.F } \\
* * * \text { corresponding layout of Figure } 3 & \end{array}$} \\
\hline
\end{tabular}

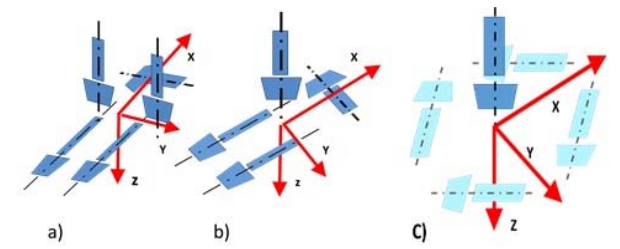

Figure 3 Examples of AUV propulsion system layout.

For this kind of applications, the usage of pivoted thrusters should be a cheap and reliable solution, which are adopted in some innovative commercial products such as the Italian SeaStick [13], which is visible in Figure 4.

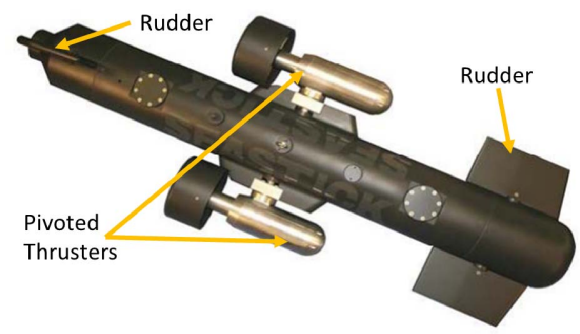

Figure 4 Examples AUV-ROV with pivoted Thrusters [14].

In addition, pivoted thrusters have been recently used for research oriented vehicles such as the Smart E, developed by University of Luebeck, where three pivoted thrusters are used to control the six degree of freedom of spherical/saucer AUV[1],[2].

\section{DESIGN OF PIVOTED Thruster}

Each pivoted thrusters, visible in Figure 5, is designed as a modular and independent units composed by an oil compensated thruster, a servomotor, a magnetic joint, some modular fixture elements and the oil compensation system.

The oil compensated thruster, was internally developed and identified by authors and is the same, which is used for the propulsion of MARTA [6] and Feel Hippo AUVs.

The servomotor controls the angular position of the thruster with respect to the hull frame. In this way, the module is able to define the angular orientation of the thruster. In addition, in this case the servomotor, as the thruster, is oil filled in order to assure a good reliability with respect to different operating depths. 


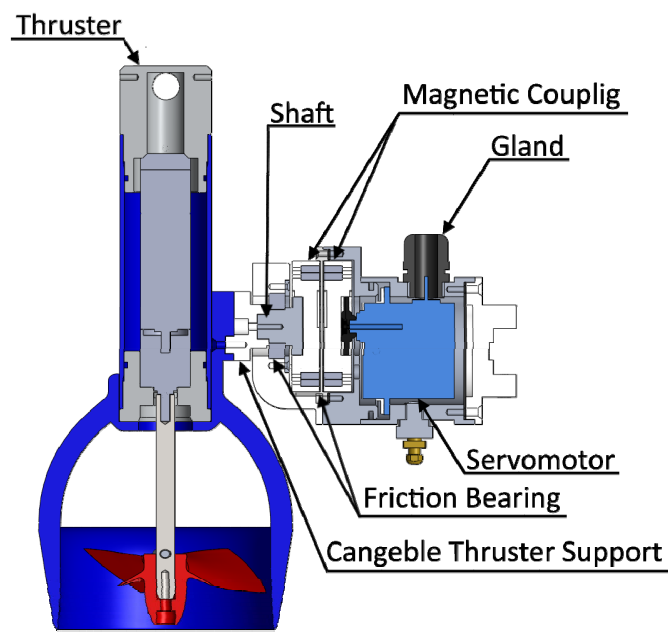

Figure 5 Proposed pivoted thruster.

The pressure compensation system consists in three simple component: the oil refill system, two compensation holes, and a compensation shell (Figure 6). The oil refill system allows the filling of the servomotor case with the oil without air. The compensation holes and compensation shell allows to transfer the pressure of external water to the oil. This system allows the use of this pivoted thruster even at high depths.
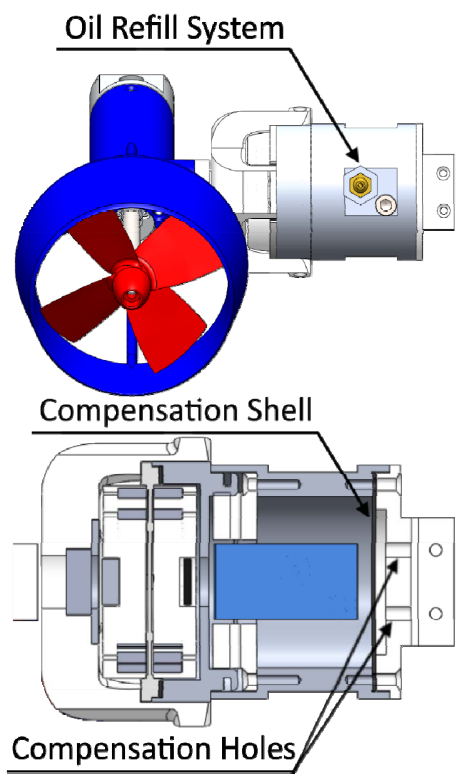

Figure 6 Pivoted Thruster, detail of the pressure compensation system

Authors chose a magnetic coupling to connect the servomotor to the thruster in order to increase the reliability of the system against torque overload and water contamination. Finally, some additional modular fixtures are designed, in order to easily customize the relative orientation of the thruster with respect to its pivoting axis and respect to the hull.

For this activity, the most interesting things are related to the design of the magnetic coupling and more generally to the design of the sealed case of the position controlled motors, which is described in detail in Figure 5.

\section{MAGNETIC COUPLIG}

Magnetic couplings and gears are used for applications involving low mechanical losses, maintenance-free operations, and inherent overload protection. In addition, for marine and hydraulic applications, the indirect coupling assured by magnetic joints is useful to reduce the risk of contamination of components like motors, which cannot be directly exposed to the operated fluid.

As visible in Figure 7, in order to couple the servomotor to the thruster, it is possible to use both radial and axial magnetic joints. In this work, after a preliminary design, it was preferred an axial solution in order to reduce the radial encumbrances.

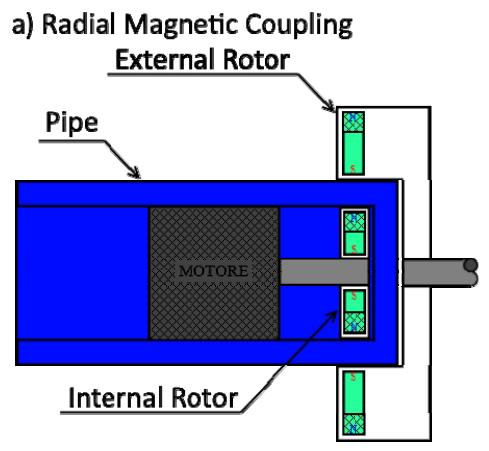

b) Axial Magnetic Coupling

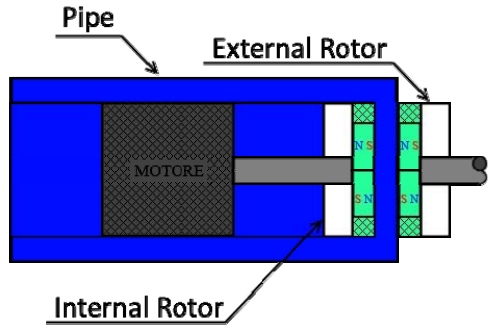

Figure 7 Type of magnetic couplings.

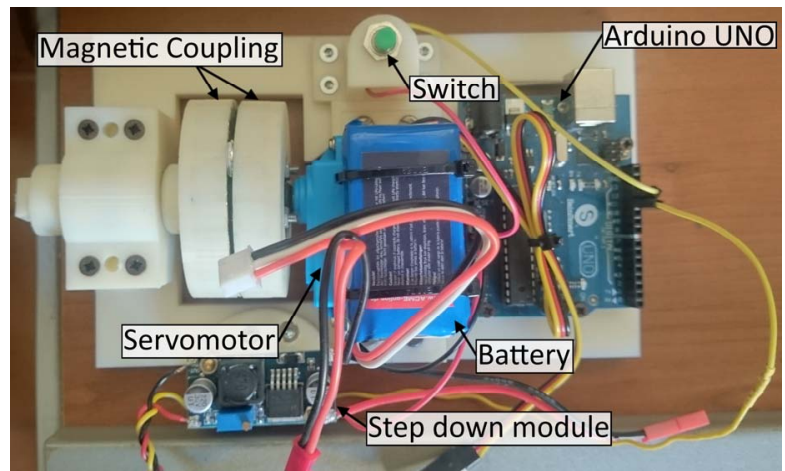

Figure 8 Experimental layout adopted for testing the magnetic coupling.

Authors designed the joint with the aid of a mathematical model, based on the theoretical and empirical relationships developed by Furlani [15], [16]. The goal of the design has been the realization of a small and economic magnetic coupling able to convey at most $75 \%$ of max torque of the servomotor, in order to protect the motor from overload. These constrains led to realize a joint composed by a large number of 
small magnets, with a diameter of $57 \mathrm{~mm}$ and with the capability to convey about $1 \mathrm{Nm}$ of torque. The performances of this joint was verified by using the experimental test rig, as visible in Figure 8. A mechanical interface was used to apply a known measured torque.

\section{PRELIMINARY IDENTIFICATION OF PROPELLER BEHAVIOR TO FOUR QUADRANTS OPERATION}

For a proper design of the whole propulsion system, the behavior of the thruster had to be identified. Adopted thrusters is composed by a standard $\mathrm{Ka} \mathrm{4-70} \mathrm{propeller} \mathrm{ducted} \mathrm{with} \mathrm{a} \mathrm{19-}$ A convergent nozzle with a $\mathrm{p} / \mathrm{d}$ ratio of 1.4 and a diameter of $90 \mathrm{~mm}$. Since the same thruster has been previously adopted for the propulsion of MARTA, authors have some data concerning bollard thrust tests, executed in the pool of MDM lab (Pistoia Italy), and concerning the performances of the thruster [6]. Results provided preliminary identification of the propeller from with it is possible to extrapolate its behavior to simulate four quadrants operation.

The study of four quadrants operation of the propeller is quite difficult with respect of the study of the simple propeller advancing movement. This difficulties are related to the possible zero value or the sign inversions of advance speed $\left(\mathrm{V}_{\mathrm{a}}\right.$ $[\mathrm{m} / \mathrm{s}]$ ), so the rotational speed (n $[\mathrm{Hz}]$ ) leads to a numerically inconsistent description of most commonly used parameters as advance coefficient $(\mathrm{J})$, thrust and torque coefficients $\left(\mathrm{K}_{\mathrm{T}}\right.$ and $K_{Q}$ ) [17], [18]. For this reason, propellers advance is expressed in terms of advance angle $\beta$ (defined according (1)), where the advance speed $\mathrm{V}_{\mathrm{a}}$ is scaled with respect to the propeller tangential speed calculated at the $70 \%$ of the propeller tip radius:

$$
\beta=\operatorname{atan} \frac{V_{a}}{0.7 \pi n D}
$$

By defining the $\beta$ parameter, is possible to redefine thrust and torque coefficients. New parameters are respectively named $\mathrm{C}_{\mathrm{T}}$ (2) and $\mathrm{C}_{\mathrm{Q}}$ (3) and are respectively defined as thrust (T) and torque (Q), scaled with respect to kinetic energy associated to the inlet relative speed also referred to the $70 \%$ of the propeller tip radius:

$$
\begin{aligned}
& C_{T}=\frac{T}{(\pi / 8) \rho\left[V_{a}^{2}+(0.7 \pi n D)^{2}\right] D^{2}} \\
& C_{Q}=\frac{Q}{(\pi / 8) \rho\left[V_{a}^{2}+(0.7 \pi n D)^{2}\right] D^{3}}
\end{aligned}
$$

$\mathrm{C}_{\mathrm{T}}$ and $\mathrm{C}_{\mathrm{Q}}$ coefficients are typically approximated in terms of Fourier series (4), (5).

$$
C_{T}=\sum_{k=0}^{20} A_{k} \cos (k \beta)+B_{k} \sin (k \beta)
$$

$$
C_{Q}=\sum_{k=0}^{20} C_{k} \cos (k \beta)+D_{k} \sin (k \beta)
$$

For the chosen propeller Ka 4-70 coefficients $A_{k}, B_{k}, C_{k}$, and $D_{k}$ are known from literature [19], [20] and experimentally verified, by the authors [6].

In particular, Figure 9 shows the behavior of the modified coefficient $\mathrm{C}_{\mathrm{T}}$ with respect to $\beta$ : results of bollard thrust tests are obtained with values of $\beta$ equal to 0 (forward thrust) and $\pi$ (reversed thrust).

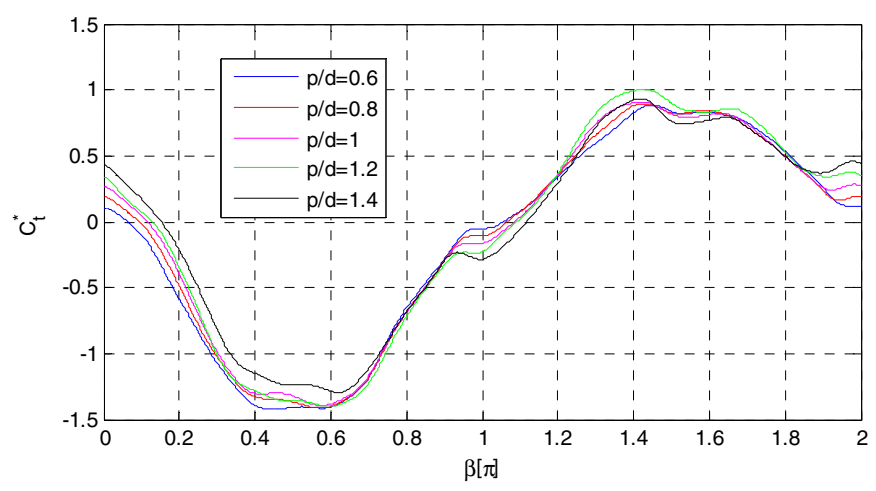

Figure $9 C_{t}$ for a Ka 4-70 Propeller ducted on a 19-A nozzle, according Oerstveld [19].

\section{DEVELOPMENT OF A VIRTUAL MODEL OF THE VEHICLE}

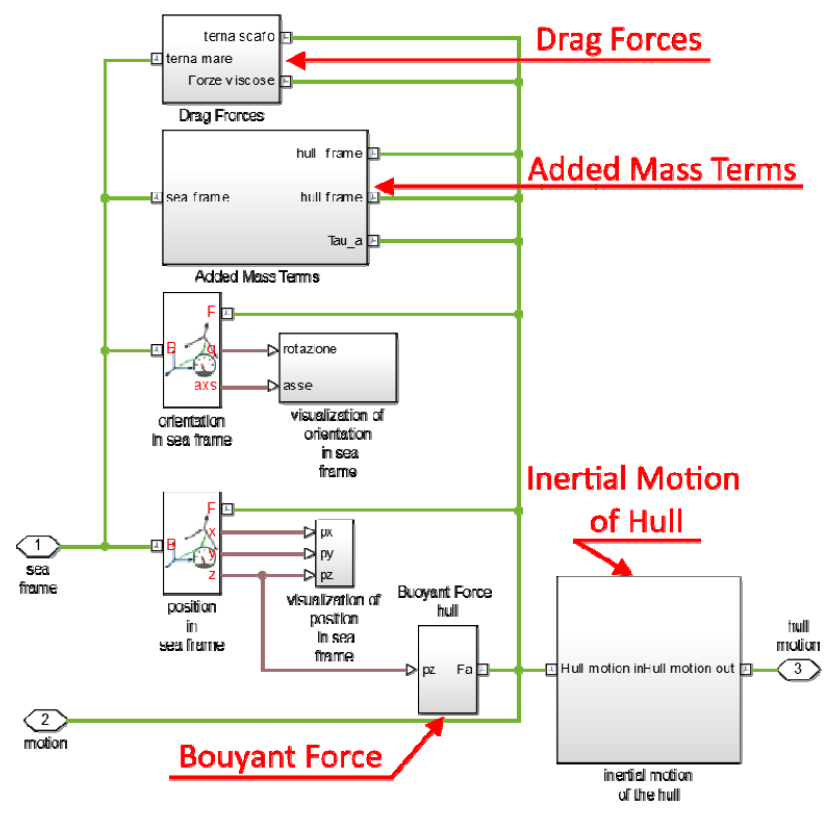

Figure 10 Example of a sub-module of Simscape model.

Authors developed a virtual model of the vehicle in the Simscape environment of Matlab ${ }^{T M}$ to test the new vehicle layout, visible in Figure 1, and compare it with the original MARTA layout. In particular, authors use the model of original 
MARTA layout as a benchmark/reference test case. Models are organized in modular way, so both models was assembled using almost the same set of Simscape blocks and functions. Furthermore, it was necessary realized customs library to calculate hydrodynamics force, according relations defined in literature [8] and to define the control system of the vehicle. An example of the result of this process is visible in Figure 10.

In order to control the vehicles to tests their performances, authors had to define an appropriated control logic. For this application, authors chosen for both models a decoupled control. This control solution is based on SISO (single input single output) PID (Proportional-Integral-Derivative) regulator, which control independently each degree of freedom of the hull. The six reference commands generate by the controller, are converted in the corresponding action requested to actuators, by an allocation matrix. In particular, for pivoted layout the allocation matrix correspond to $8 \times 6$ matrix, as visible in equation (6):
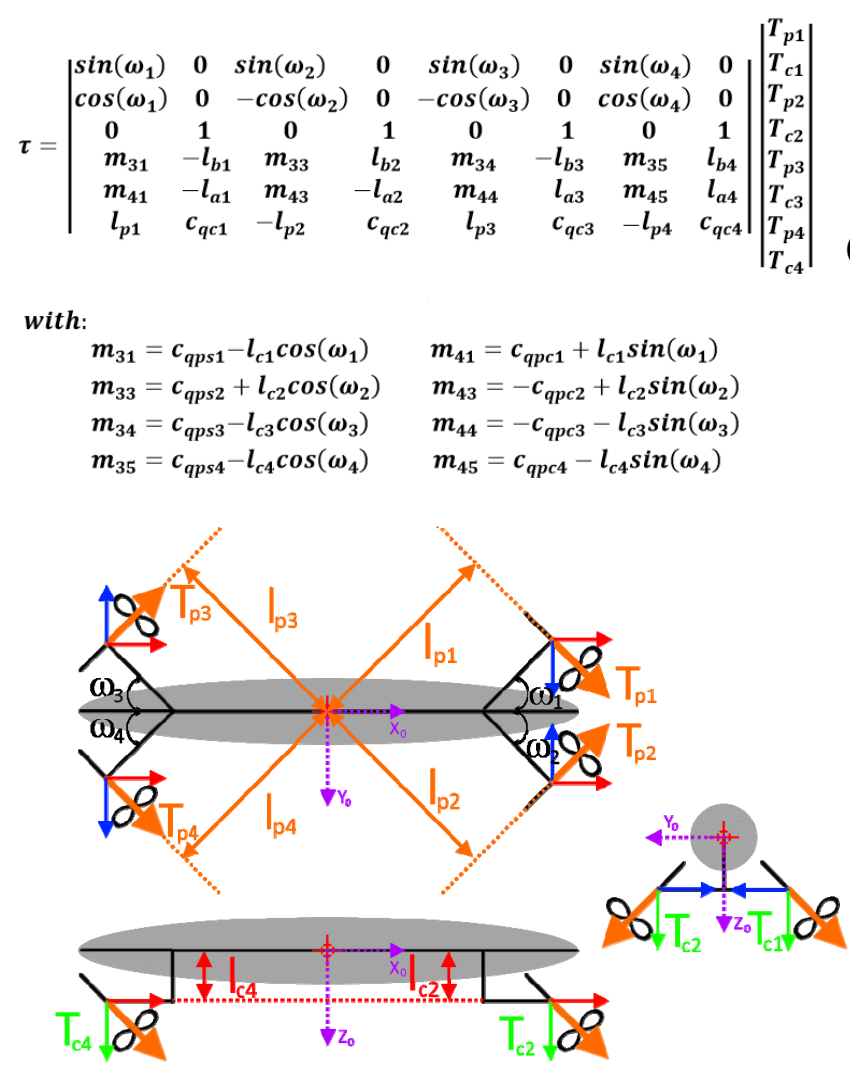

Figure 11 Scheme of pivoted layout.

The parameters in relation (6) are shown in Figure 11, and $\mathrm{c}_{\mathrm{qps} 1}, \mathrm{c}_{\mathrm{qps} 2}, \mathrm{c}_{\mathrm{qps} 3}, \mathrm{c}_{\mathrm{qps} 4}, \mathrm{c}_{\mathrm{qpc} 1}, \mathrm{c}_{\mathrm{qpc} 2}, \mathrm{c}_{\mathrm{qpc} 3}, \mathrm{c}_{\mathrm{qpc} 4}, \mathrm{c}_{\mathrm{qc} 1}, \mathrm{c}_{\mathrm{qc} 2}, \mathrm{c}_{\mathrm{qc} 3}, \mathrm{c}_{\mathrm{qc} 4}$ are parameters necessary to model effects of torques generated by propellers on motors axes.

Obviously, the allocation matrix generate eight components of motor actions, so it is necessary to define the corresponding four motor thrusts and four motor orientation angles. In this application, authors decided to obtain motors angles as arctangent of the ratio between corresponding $T_{p}$ e $T_{c}$ for each motor. Instead, motors thrusts are calculated as the module of the corresponding vector $\left|T_{p} T_{c}\right|^{T}$.

\section{RESULTS}

In order to compare the maneuverability of two examined vehicles, authors had to define an appropriate parameter named maneuverability. This parameter was defined as the inverse of the power consumption of motors normalized with respect to third power of the velocity of the vehicle (7). Finally, to realize a graphic representation of vehicles maneuverability in the horizontal plane, authors decided to represent results in a polar graphics, where the maneuverability was compared respect the direction of the velocity.

$$
\text { Maneuverability }=\frac{\text { Velocity }^{3}}{\text { Power Consumption }}
$$

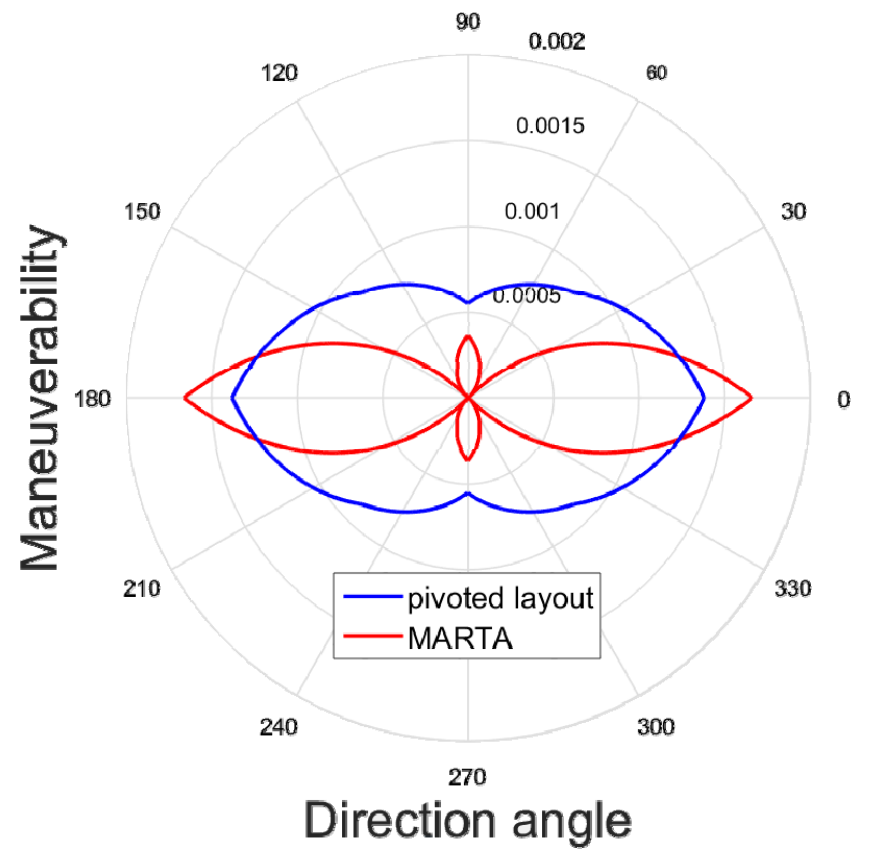

Figure 12 Comparison between maneuverability of pivoted layout of the vehicle and maneuverability of MARTA.

As visible in Figure 12, the maneuverability of pivoted layout with respect to the maneuverability of original MARTA layout is extremely increased. This condition it is due to the better use of motor in the pivoted configuration. In fact, the pivoted layout vehicle exploits constantly all thrusters simultaneously, whereby all motor running at low RPM. Conversely, MARTA can use only two thrusters to realize a single motion, whereby motors had to run at higher RPM, to push the vehicle at the same velocity of the proposed layout. Unfortunately, the improved motion capability involves a loss in maneuverability for what concerns advance motion, but the reduction of maneuverability is only about $17 \%$, so it is not a penalizing condition. However, it is possible to tune this behavior, because the front section is related to $\omega_{1}, \omega_{2}, \omega_{3}$ and $\varpi_{4}$ angles (Figure 11). The comparison graph, shown in Figure 
12 , was computed with these angles equal to $45^{\circ}$, so reducing values of these angles, the maneuverability in forward direction increases and consequently lateral moving capability is reduced. Conversely, if values of angles decrease, lateral motion performances are privileged respect to longitudinal ones. It is interesting to observe that this behavior can be exploited to properly tune the vehicle with respect to the operating conditions.

Another interesting comparison is related to motions in the vertical direction. In this case, buoyancy forces and structural differences between two layouts heavily influence the comparison. This particular condition entail that the maneuverability of two layouts are comparable, when vehicles go down, but when vehicles go up, the maneuverability of pivoted layout is about 36 time better than MARTA. This condition is, probably, due to the better stability and capability to maneuver of the proposed layout. Indeed the motion of MARTA involve additional loss of efficiency of tunneled thrusters, which turn to very high RPM to provide adequate motion corrections.

The pivoted layout not only offers improved maneuverability but also confers enhanced failure robustness. Indeed, three pivoted thrusters are enough to perform motion in 6 degrees of freedom. With the proposed layout, in case of motor failure or servomotor failure, the vehicle can move in an acceptable way, without losing any degree of freedom. Of course, it is necessary to adopt a modify control approach. For example, if the vehicle has a failure of a single motor, it is possible remove two columns, which represent the corresponding contributions in the matrix. An example is shown in (8), where the authors supposed to have a failure of the fourth motor module.

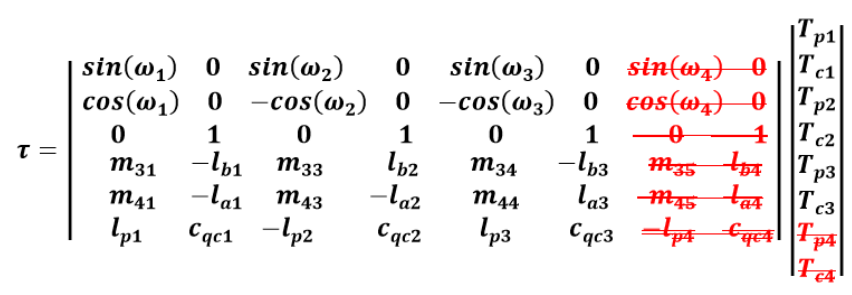

Obviously, in these cases, the maneuverability of the vehicle decrease, but the AUV, keeps sufficient operating capability to complete the mission or to safely return to the base.

\section{CONCLUSIONS}

In conclusion, proposed vehicle layout results more efficient with respect to the most common layouts, generally adopted in ROV and AUV. For this reason, this solution allows building vehicles with reduced power consumption, thus the mission length can grow. In addition, pivoted thrusters increase the maneuverability of the vehicle, allowing to perform more complex path with the AUV. Finally, the use of four pivoted thrusters on a vehicle, make it possible to successfully face different motor failures, although increase the complexity of control matrices.

\section{REFERENCES}

[1] B. Meyer, K. Ehlers, C. Osterloh, E. Maehle1, Smart-E An Autonomous Omnidirectional Underwater Robot, Paladyn, Journal of Behavioral Robotics. Volume 4, Issue 4, Pages 204-210, ISSN (Print) 2081-4836, DOI: 10.2478/pjbr-2013-0015, December 2013.

[2] Ehlers, K., Meyer, B., Maehle, E.,Full holonomic control of the omnidirectional AUV SMART-E, (2014) Proceedings for the Joint Conference of ISR 2014 - 45th International Symposium on Robotics and Robotik 2014 - 8th German Conference on Robotics, ISR/ROBOTIK 2014, pp. 299-304.

[3] B.Allotta, L.Pugi, F. Bartolini, A.Ridolfi, R. Costanzi, N.Monni, J.Jelli. Preliminary design and fast prototyping of an Autonomous Underwater Vehicle propulsion system, Proceedings of the Institution of Mechanical Engineers, Part M: Journal of Engineering for the Marit October 6, 2015 0: 1475090215605133v1-1475090215605133

[4] Allotta, B., Costanzi, R., Monni, N., Pugi, L., Ridolfi, A., Vettori, G. Design and simulation of an autonomous underwater vehicle (2012) ECCOMAS 2012 - European Congress on Computational Methods in Applied Sciences and Engineering, e-Book Full Papers, pp. 3656-3673.

[5] Allotta B, Pugi L, Costanzi R, et al. Localization algorithm for a fleet of three AUVs by INS, DVL and range measurements. In: Proceedings of the 15th international conference on advanced robotics, 2011, Tallinn, 20-23 June 2011, pp.631-636. New York: IEEE.

[6] B. Allotta, R. Costanzi, J.Gelli, L. Pugi, A. Ridolfi, Design of a Modular Propulsion System for MARTA AUV, In proceedings of OCEANS'15 MTS/IEEE GENOVA. IEEE, ISBN: 978-1-4799-8736-8, Genova, Italia, 18-21 maggio 2015.

[7] Official Web-Site of the ARROWS Project (ARcheological Robot systems for the World's Seas) http://www.arrowsproject.eu/

[8] Fossen, T. I. (1994). Guidance and Control of Ocean vehicles, Wiley, ISBN 0-471-94113-1.

[9] Curtis TL, Perrault D, Williams C, et al. C-SCOUT: a general-purpose AUV for systems research. In: Proceedings of the 2000 international symposium on underwater technology 2000 (UT '00), Tokyo, Japan, 23-26 May 2000, pp.73-77. New York: IEEE.

[10] Stokey RP, Roup A, von Alt C, et al. Development of the REMUS 600 autonomous underwater vehicle. In: Proceedings of MTS/IEEE OCEANS, 2005, Washington, DC, 17-23 September 2005, vol. 2, pp.1301-1304. New York: IEEE.

[11] Whitney, J. W., and S. M. Smith. "Observations on the dynamic performance of tunnel thrusters." OCEANS'98 Conference Proceedings. Vol. 2. IEEE, 1998.

[12] A B Phillips, L. Steenson, C. Harris, E.Rogers, S. R. Turnock, M. Furlong, Delphin2: An over actuated autonomous underwater vehicle for manoeuvring research,Trans RINA, Vol 151, Part A1, Intl J Maritime Eng, 2009 Jan-Mar.

[13] A. Alvarez, A. Caffaz, A. Caiti, G. Casalino L. Gualdesi, A. Turetta, R Viviani, Folaga: A low-cost autonomous underwater vehicle combining glider and AUV capabilities, Ocean Engineering 36 (2009) 24-38 A.

[14] A. Faccioli, SeaStick AUV con funzionalità ROV, proceedings of Operational Oceanography, Innovative technologies and applications, 3-5June 2013 Oristano, Italy.

[15] Furlani, Edward P. Permanent magnet and electromechanical devices: materials, analysis, and applications. Academic Press, 2001.

[16] Furlani, E. P. "A two-dimensional analysis for the coupling of magnetic gears." IEEE Transactions on Magnetics 33.3 (1997): 2317-2321.

[17] Carlton J. Marine propellers and propulsion. 2nd ed. Elsevier, 2007.

[18] Pivano L, Johansen TA and Smogeli ØN. A four quadrant thrust estimation scheme for marine propellers: theory and experiments. IEEE T Contr Syst T 2009; 17(1): 215-226.

[19] Oosterveld MWC. Wake adapted ducted propellers. Wageningen: Netherlands Ship Model Basin (NSMBPublication No. 345), 1970.

[20] Oosterveld MWC. Ducted propeller characteristics. In: RINA symposium on ducted propellers, London, 1973. 